\title{
Beclin1 and HMGB1 ameliorate the a-synuclein-mediated autophagy inhibition in PC12 cells
}

Kaihua Wang ${ }^{1 \dagger}$, Jianmin Huang ${ }^{1 \dagger}$, Wei Xie ${ }^{2,3,4^{*}}$, Longjian Huang ${ }^{1}$, Canhua Zhong ${ }^{1}$ and Zhenzhen Chen ${ }^{5}$

\begin{abstract}
Background: Aberrant a-synuclein aggregation due to the deficiency of ubiquitin-proteasome or of autophagy characterizes the parkinson disease (PD). High mobility group box 1 (HMGB1) is a novel stress sensor to mediate the persistent neuro-inflammation and the consequent progressive neurodegeneration, via controlling the cellular autophagy/apoptosis checkpoint during inflammation. Moreover, HMGB1 has been recently indicated to involve in the autophagic degradation of a-synuclein.

Methods: In the current study, we investigated the influence of the overexpressed a-synuclein of wild type (wt) or mutant type (A53T and A30P, $\mathrm{mt}$ ) on the cytosolic levels of HMGB1 and Beclin1 and on the starvation-induced autophagy in pheochromocytoma PC12 cells. And then we explored the overexpression of HMGB1 or of Beclin1 on the a-synuclein degradation and on the autophagy in the a-synuclein-overexpressed PC12 cells.

Results: It was demonstrated that a-synuclein overexpression inhibited the trans-location of HMGB1 from nucleus to cytosol and reduced the cytosolic level of Beclin1 in PC12 cells, and inhibited the starvation-induced autophagy via downregulating autophagy-associated markers and via reducing the autophagic vesicles in PC12 cells under starvation. On the other side, the intracellular promotion of either HMGB1 or Beclin1 upregulated the a-synuclein degradation and ameliorated the a-synuclein-mediated autophagy reduction in PC12 cells. However, the exogenous HMGB1 treatment exerted no such regulation in PC12 cells.

Conclusion: In summary, our study confirmed the positive regulation by HMGB1 and Beclin1 on the a-synuclein degradation and on the starvation-induced autophagy in PC12 cells, implying both markers as prominent targets to promote the a-synuclein degradation.
\end{abstract}

Keywords: Beclin1, HMGB1, a-synuclein, Autophagy, Pheochromocytoma PC12 cells

\section{Background}

Aberrant $\alpha$-synuclein aggregation and the followed degeneration of dopaminergic cells [1-4] characterize the parkinson disease (PD). The deficient protein degradation pathways such as ubiquitin-proteasome system (UPS) and autophagy lysosomal pathway (ALP) have been recognized to contribute to the scanty degradation of wild-type (WT) or mutant-type (MT, A53T and A30P) $\alpha$-synuclein $[5,6]$.

\footnotetext{
* Correspondence: weixiesmu@sina.com

${ }^{\dagger}$ Equal contributors

${ }^{2}$ Southern Medical University, Guangzhou, Guangdong 510515, China

${ }^{3}$ Department of Traditional Chinese Medicine, Nanfang Hospital, Southern

Medical University, Guangzhou, Guangdong 510515, China

Full list of author information is available at the end of the article
}

Moreover, autophagy is the only clearance pathway for the aggregated $\alpha$-syn $[7,8]$. And the both macromolecular complexes, mammalian target of rapamycin (mTOR)Unc-51 like autophagy activating kinase 1 (ULK1) complex and the Beclin 1-Phosphatidylinositol 3-kinase catalytic subunit type 3 (PIK3C3) complex [9] are important for $\alpha$-synuclein degradation [10].

Recently, multiple molecules have been characterized to regulate autophagy via the mTOR-ULK1 or PIK3C3 pathway. Amino acid starvation stimulates phosphorylation of ULK1 by protein phosphatase $2 \mathrm{~A}$ to form PP2A-B55 $\alpha$ complex and induces the ULK1-dependent autophagy [11, 12]. The transcriptional regulation of Annexin A2 also promotes the starvation-induced 
autophagy, via regulating autophagosome formation by enabling appropriate Autophagy-related protein (ATG) 9A trafficking from endosomes to autophagosomes [13]. Moreover, various markers have been confirmed to regulate autophagy through the PIK3C3 pathway. Dapper1 promotes autophagy by enhancing the Beclin1- vacuolar protein sorting 34 (VPS34)-Atg14L complex formation [14]. Interferon-alpha-2b induces autophagy in hepatocellular carcinoma cells via stimulating Beclin1 pathway [15]. Rho-associated, coiled-coil containing protein kinase 1 (ROCK1) promotes autophagy by binding and phosphorylating Beclin1 at Thr119 under metabolic stress [16]. However, Macrophage stimulating 1 (Mst1) inhibits autophagy by promoting the interaction between Beclin1 and Bcl-2 [17]. And Smad2 binds to the Beclin1 promoter region, transcriptionally regulates autophagy via regulating beclin1 expression [18].

High mobility group box 1 (HMGB1) is a stress sensor that plays a critical role in various physiological and pathological processes including cell development, differentiation, inflammation, metabolism and death [19]. Increasing evidence demonstrates that HMGB1-dependent autophagy promotes chemotherapy resistance [20], sustains tumor metabolism [21], protects against endotoxemia [22] and involves in other pathological processes $[23,24]$. Inflammation has also been recognized to involve in PD [25-27]. Sustained inflammatory process and the activated microglia might drive the progressive degeneration of dopamine neurons in PD [28]. Moreover, the cytoplasmic HMGB1 has been indicated to control the cellular autophagy/apoptosis checkpoint during inflammation [29]. HMGB1 was demonstrated to mediate the persistent neuroinflammation and consequent progressive neurodegeneration, via stimulating the production of multiple inflammatory and neurotoxic factors [30]. In addition, HMGB1 has been recently indicated to involve in the autophagy inhibition caused by $\alpha$-synuclein overexpression [31], implying a direct role in modulating autophagic degradation of $\alpha$-synuclein.

In current study, we investigated the regulation of $\alpha$ synuclein overexpression on the trans-location or the expression of HMGB1 and Beclin1, and on the starvationinduced autophagy in pheochromocytoma PC12 cells. And then we explored the overexpression of HMGB1 or Beclin1 on the $\alpha$-synuclein degradation and on the autophagy in the $\alpha$-synuclein-overexpressed PC12 cells. This study confirmed the positive regulation by endogenous HMGB1 on the autophagy-mediated $\alpha$-synuclein degradation in PC12 cells.

\section{Methods}

\section{Construction of PC12 $\left(\right.$ Syn $\left.^{\text {wt }}\right)$ and PC12 $\left(\mathrm{Syn}^{\mathrm{mt}}\right)$ cells}

Rattus norvegicus pheochromocytoma PC12 cell line was purchased from American Type Culture Collection
(ATCC) (Rockville, MD, USA) and was cultured in RPMI-1640 medium (GIBCO, Rockville, MD, USA) supplemented with $10 \%$ (2 \% for maintaining) fetal bovine serum (FBS) (Hyclone, Pittsburgh, PA, USA) and with $100 \mathrm{U} / \mathrm{mL}$ penicillin and $100 \mathrm{mg} / \mathrm{mL}$ streptomycin (CSPC Pharmaceutical Group Limited, China). Cells were cultured at $37{ }^{\circ} \mathrm{C}$ in a humidified incubator with $5 \% \mathrm{CO}_{2}$. To construct an $\alpha$-synuclein-overexpressed PC12 cell line, the wild-type (wt) (NM_019169.2), mutanttype (mt, A53T and A30P) $\alpha$-synuclein coding sequence, or the Enhanced Green Fluorescence Protein (EGFP) coding sequence (as control) was amplified and cloned into the pcDNA3.1(+) vector (Invitrogen, Carlsbad, CA, USA). Then we transfected the $\alpha$-Syn $(w t)-p c D N A 3.1(+), \alpha-$ Syn $(m t)-p c D N A 3.1(+)$ or EGFP-pcDNA3.1(+) plasmid into the $85 \%$ confluent PC12 cells with lipofectamine 2000 (Invitrogen, Carlsbad, CA, USA). After the transfection for 24 hours, cells were updated with the RPMI-1640 medium which were supplemented with $10 \% \mathrm{FBS}$ and with $1.2 \mathrm{mg} / \mathrm{ml}$ G418 (Life Technologies, Grand Island, NY, USA) to select the cell clone overexpressing wt or $\mathrm{mt}$ $\alpha$-synuclein or EGFP. And the selected PC12 $\left(\operatorname{Syn}^{\mathrm{wt}}\right)$, PC12 (Syn ${ }^{\mathrm{mt}}$ ) and PC12 (Con) cell clones were cultured in RPMI-1640 medium $+10 \%$ FBS supplemented with $0.8 \mathrm{mg} / \mathrm{ml} \mathrm{G} 418$. For the starvation treatment, PC12 $\left(\mathrm{Syn}^{\mathrm{wt}}\right), \mathrm{PC} 12\left(\mathrm{Syn}^{\mathrm{mt}}\right)$ and PC12 (Con) cells were inoculated with FBS-free RPMI-1640 medium at $37{ }^{\circ} \mathrm{C}$ in a humidified chamber with $5 \% \mathrm{CO}_{2}$.

\section{Overexpression of HMGB1 or Beclin 1 in PC12 (Syn ${ }^{\text {wt }}$ ) or PC12 $\left(\mathrm{Syn}^{\mathrm{mt}}\right)$ cells}

To promote the HMGB1 level in PC12 $\left(\mathrm{Syn}^{\mathrm{wt}}\right)$ or PC12 $\left(\mathrm{Syn}^{\mathrm{mt}}\right)$ cells, the coding sequence of HMGB1 or the coding sequence of Red Fluorescence Protein (RFP) was cloned into the pcDNA3.1(+) vector. And $5 \mathrm{~g} / \mathrm{mL}$ (to guarantee more than $90 \%$ cells to be transfected) HMGB1-pcDNA3.1(+) or RFP-pcDNA3.1(+) plasmid was transfected into $10^{5}$ per well $85 \%$ confluent PC12 $\left(\mathrm{Syn}^{\mathrm{wt}}\right)$ or PC12 $\left(\right.$ Syn $\left.^{\mathrm{mt}}\right)$ cells. To upregulate the Beclin1 level in PC12 cells, Beclin1 or the coding sequence of Chloramphenicol acetyl transferase(CAT) was amplified and was cloned into the pLenti 6/TR vector (Invitrogen, Carlsbad, CA, USA). Recombinant pLenti-Beclin1 (LV-Beclin1) or pLenti-CAT virus of (LV-Con) was produced by cotransfecting 293 T cells with pLenti-Beclin1 or pLenti-CAT and ViraSafe $^{\text {mix }}$ Lentiviral Packaging System (Cell Biolabs, San Diego, CA, USA). PC12 (Syn $\left.{ }^{\mathrm{wt}}\right)$ or PC12 $\left(\mathrm{Syn}^{\mathrm{mt}}\right)$ cells were infected with LV-Beclin1 or LV-Con virus with 1 Multiplicity of infection (MOI) for 12 or 24 hours.

\section{mRNA isolation and real-time PCR analysis of a-synuclein mRNA}

mRNA samples from OVCAR-3 cells were prepared with Kit for mRNA Isolation and Purification (Clontech, 
Palo Alto, CA, USA), and reverse transcription (RT) was performed with M-MLV Reverse Transcriptase (Promega, Madison, WI, USA) as following: $42{ }^{\circ} \mathrm{C}$ for $5 \mathrm{~min}$ and $95^{\circ} \mathrm{C}$ for $10 \mathrm{sec}$ for the reverse transcription. And the real-time PCR assay was performed at $95{ }^{\circ} \mathrm{C}$ for $5 \mathrm{sec}$ and $60^{\circ} \mathrm{C}$ for $20 \mathrm{sec}$ for the PCR reaction, with 40 cycles. The primer sequences for $\alpha$-synuclein were as following: forward primer, 5'- CGT CCT CTA TGT AGG TTC CA -3', reverse primer: 5'- GCC ACT GTT GTC ACT CCA TG -3'. The primer sequences for -actin were as following: forward primer, 5'- GTA CCC TGG CAT TGC CGA CA -3, reverse primer: 5'- GGA CTC GTC ATA CTC CTG CTT GCT -3'. Relative $\alpha$-synuclein mRNA level was normalized to internal control -actin, with the $\Delta \Delta \mathrm{Ct}$ method [32].

\section{Western blotting assay}

PC12, PC12 (Con), PC12 (Syn $\left.{ }^{\mathrm{wt}}\right)$ or PC12 $\left(\mathrm{Syn}^{\mathrm{mt}}\right)$ cells were homogenized with an ice-cold NE-PER Nuclear and Cytoplasmic Extraction Reagents Kit (Pierce, Rockford, IL, USA), the nuclear and the cytoplasmic proteins were isolated according to the product's manual. Each protein sample was supplemented with a protease Inhibitor Cocktail (Abcam, Cambridge, UK) and was stored at $-80{ }^{\circ} \mathrm{C}$ before use. The western blotting assay was performed with the rabbit polyclone antibody against $\alpha$-synuclein (Abcam, Cambridge, UK), against HMGB1(Abcam, Cambridge, UK), against Beclin1 (Cell Signaling Technology Inc., Danvers, MA, USA), against LC3 (Pierce, Rockford, IL, USA), Atg 7 (Sigma-Aldrich, St. Louis, MO, USA), against mTOR (Abcam, Cambridge, UK), against LMNB1 (Pierce, Rockford, IL, USA) or against -actin (Sinobio, Beijing, China), and with horseradish peroxidase-linked secondary antibodies (Jackson ImmunoResearch, West Grove, PA, USA). The specific binding band was scanned and quantified according to the band density.

\section{Quantitative EGFP-LC3 analysis and electron microscopy} $1 \times 10^{5}$ cells/well PC12, PC12 (Con), PC12 $\left(\mathrm{Syn}^{\mathrm{wt}}\right)$ or PC12 (Syn ${ }^{\mathrm{mt}}$ ) cells were transfected with 2 g EGFPLC3 reporter plasmid, pcDNA3.1-EGFP-LC3, with Lipofectamine 2000 (Invitrogen, Carlsbad, CA, USA) for a 24 hour's incubation to quantify the EGFP-positive autophagic vesicles which were promoted by various treatments. Then the EGFP-positive vesicles were visualized under fluorescence microscopy (JEM1230, Japan).

\section{Statistical evaluations}

Quantitative data was presented as mean \pm SE and was analyzed for a significant difference with the Student's $t$ test or one way ANOVA test. A $p$ value less than 0.05 was considered to be statistically significant.

\section{Results}

a-synuclein overexpression inhibits the trans-location of HMGB1 and the expression of Beclin1 in PC12 cells

To investigate the regulation of $\alpha$-synuclein overexpression on the trans-location or the level of HMGB1 and Beclin1, and on the starvation-induced autophagy in pheochromocytoma $\mathrm{PC12}$ cells, we constructed the PC12 cell overexpressing WT $\alpha$-synuclein, PC12 (Syn ${ }^{\text {wt }}$ ), or the PC12 cell overexpressing MT (A53T and A30P) $\alpha$-synuclein, PC12 (Syn ${ }^{\mathrm{mt}}$ ), with PC12 (Con), which overexpressed enhanced green fluorescent protein (EGFP), as control. As shown in Fig. 1a, the mRNA level of $\alpha$-synuclein was significantly higher in both $\mathrm{PC} 12$ $\left(\mathrm{Syn}^{\mathrm{wt}}\right)$ and PC12 (Syn $\left.{ }^{\mathrm{mt}}\right)$ cells, compared with the PC12 (Con) or blank PC12 cells (either $p<0.001$ for PC12 $\left(\mathrm{Syn}^{\mathrm{wt}}\right)$ or PC12 (Syn $\left.{ }^{\mathrm{mt}}\right)$ cells). And the protein level of $\alpha$-synuclein was confirmed by the western blot analysis in the PC12 $\left(\mathrm{Syn}^{\mathrm{wt}}\right)$ or PC12 $\left(\mathrm{Syn}^{\mathrm{mt}}\right)$ cells $(p<0.001$ respectively, Fig. 1b). And then we investigated the influence of $\alpha$-synuclein overexpression on the Beclin1 level and the trans-location of HMGB1 from nucleus to cytosol. Western blotting (Fig. 1c) demonstrated that the cytosolic HMGB1 was markedly upregulated, whereas was significantly downregulated in nucleus in either PC12 $\left(\mathrm{Syn}^{\mathrm{wt}}\right)$ or PC12 $\left(\mathrm{Syn}^{\mathrm{mt}}\right)$ cells $(p<0.05$ respectively, Fig. 1c). In addition the cytoplasmic Beclin1 was also examined with western blot analysis. It was indicated that the Beclin level was significantly downregulated in both PC12 (Syn $\left.{ }^{\mathrm{wt}}\right)$ and PC12 (Syn $\left.{ }^{\mathrm{mt}}\right)$ cells $(p<0.01$ respectively, Fig. 1d). Thus, we confirmed the promotion to the trans-location of HMGB1 from nucleus to cytosol, and to the cytoplasmic level of Beclin1 in PC12 cells.

\section{a-synuclein overexpression inhibits autophagy in PC12 cells}

We then examined the regulation of the overexpressed $\alpha$-synuclein on the autophagy in PC12 cells. The autophagy induction in PC12, PC12 (Con), PC12 $\left(\mathrm{Syn}^{\mathrm{wt}}\right)$ or PC12 (Syn ${ }^{\mathrm{mt}}$ ) cells subject to starvation was examined with western blotting assay and the EGFP-LC3 reporter assay. Western blotting results (Fig. 2a) demonstrated that the conversion of LC3-I to LC3-II was markedly lower in either PC12 (Syn $\left.{ }^{\mathrm{wt}}\right)$ or PC12 $\left(\mathrm{Syn}^{\mathrm{mt}}\right)$ cells, compared to the normal PC12 or the PC12 (Con) cells $(p<$ 0.01 respectively, Fig. $2 \mathrm{~b}$ ). And such reduced autophagy was also confirmed by the reduced level of Atg7 and increased level of mTOR in either PC12 $\left(\mathrm{Syn}^{\mathrm{wt}}\right)$ or PC12 $\left(\mathrm{Syn}^{\mathrm{mt}}\right)$ cells $(p<0.01$ respectively, Fig. $2 \mathrm{~b})$. Moreover, the EGFP-LC3 reporter assay also demonstrated that there were less GFP-positive autophagic puncta in either PC12 cells. Taken together, the $\alpha$-synuclein overexpression with wild or mutant type inhibits the starvationstimulated autophagy in PC12 cells. 


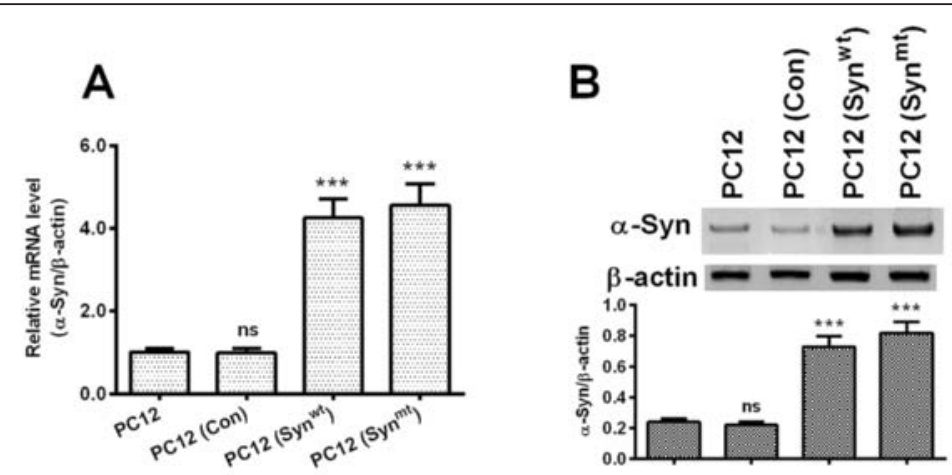

C

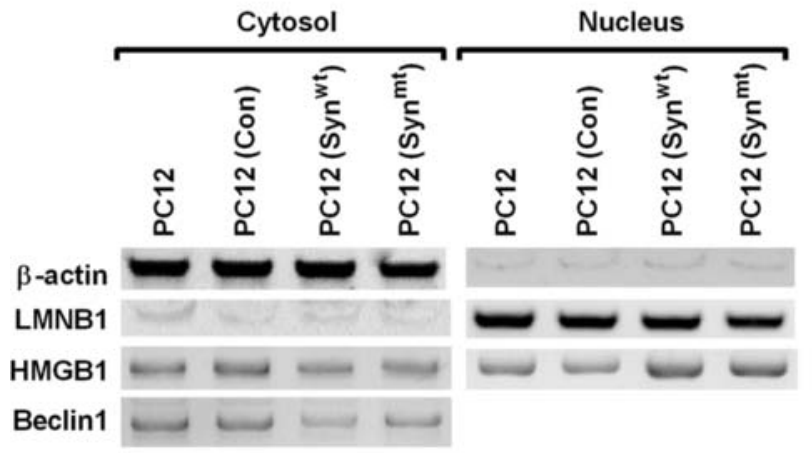

D

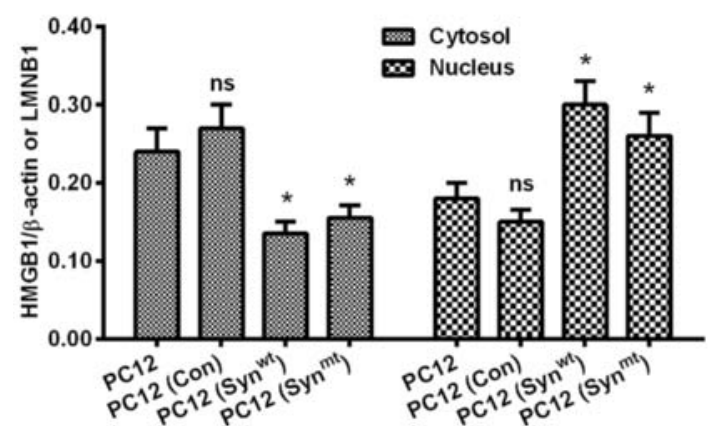

$\mathbf{E}$

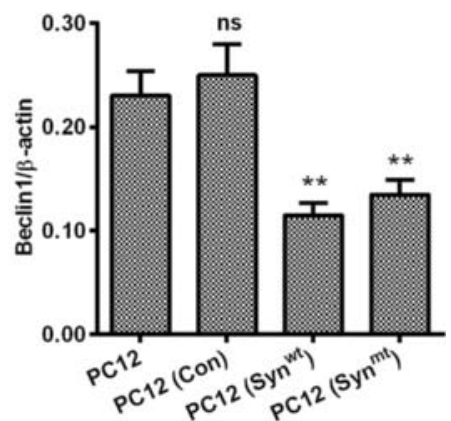

Fig. 1 Overexpression of a-synuclein with wild type (wt) or mutant type (mt) reduces cytosolic HMGB1 and Beclin1 in PC12 cells. a and $\mathbf{b}$ : mRNA (a) and protein (b) levels of a-synuclein in the PC12 cell overexpressing wt a-synuclein, PC12 (Syn ${ }^{\text {wt }}$ ), in the PC12 cell overexpressing mt (A53T and A30P) a-synuclein, PC12 (Syn ${ }^{\mathrm{mt}}$ ), in the PC12 cell overexpressing Enhanced Green Fluorescence Protein (EGFP), PC12 (Con), or in the blank P12 cells, the protein level of a-synuclein was examined by western blot analysis; c: Western blot analysis of HMGB1 (in cytosol and in nucleus) and Beclin1 (in cytosol) in PC12, PC12 (Con), PC12 (Syn ${ }^{\text {wt }}$ ) or PC12 (Syn ${ }^{\mathrm{mt}}$ ) cells; d: Relative HMGB1 level to -actin in cytosol or to Lamin B(LMNB1) in the nucleus of PC12, PC12 (Con), PC12 (Syn ${ }^{\text {wt }}$ ) or PC12 (Syn ${ }^{\mathrm{mt}}$ ) cells; e: Relative Beclin1 level to -actin in the cytosol of PC12, PC12 (Con), PC12 (Syn ${ }^{\text {wt }}$ ) or PC12 (Syn ${ }^{\mathrm{mt}}$ ) cells; Each result was averaged for triple independent experiments. Statistical significance was presented as ${ }^{*} p<0.05,{ }^{* *} p<0.01,{ }^{* * *} p<0.001$, ns: no significance

HMGB1 promotes autophagy and a-synuclein degradation in the a-synuclein-overexpressed PC12 cells

To investigate the role of HMGB1 on the autophagic degradation of $\alpha$-synuclein, we overepressed HMGB1 in both PC12 $\left(\mathrm{Syn}^{\mathrm{wt}}\right)$ and PC12 $\left(\mathrm{Syn}^{\mathrm{mt}}\right)$ cells with the gain-of-function strategy, and then re-evaluated the starvation-induced autophagy in both cell lines. Western blotting assay (Fig. 3a) demonstrated that the transfection with HMGB1-pcDNA3.1(+) (pc-HMGB1) dramatically upregulated the HMGB1 level in both PC12 (Syn ${ }^{\mathrm{wt}}$ ) (Fig. 3b) and PC12 (Syn ${ }^{\mathrm{mt}}$ ) (Fig. 3c) cells at 12 or 24 hour post transfection (H.P.T.) $(p<0.01$ or $p<0.001)$, and timedependently $(p<0.05)$. However, the $\alpha$-Synuclain level was markedly downregulated in both PC12 (Syn ${ }^{\mathrm{wt}}$ ) (Fig. 3d) and PC12 $\left(\mathrm{Syn}^{\mathrm{mt}}\right)$ (Fig. 3e) cells $(p<0.05$ respectively). Moreover, the EGFP-LC3 reporter assay (Fig. 3f) demonstrated 


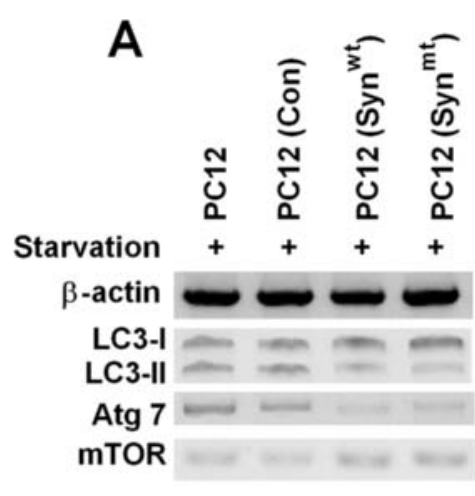

C

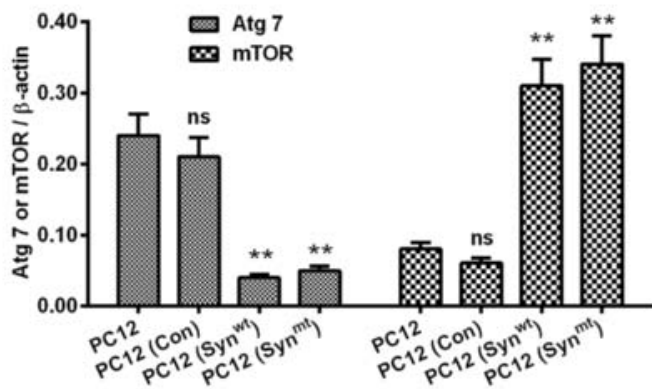

B

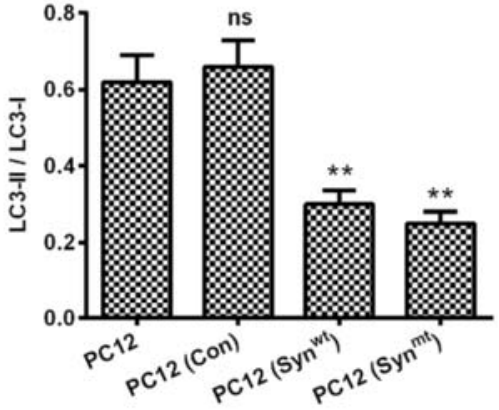

E

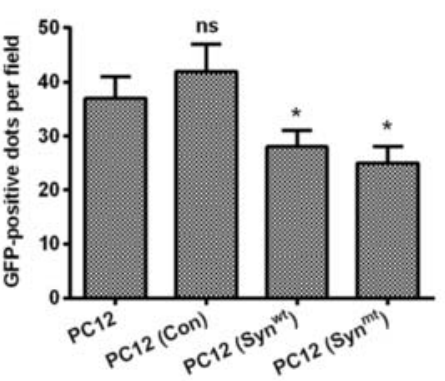

D

PC12

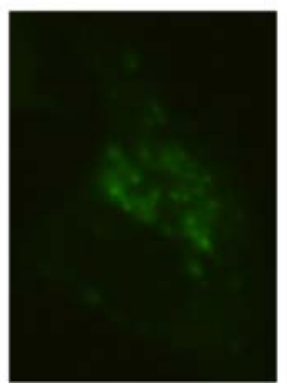

PC12 (Con)

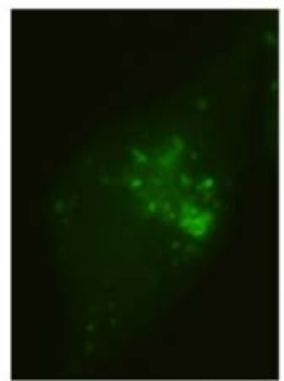

PC12 (Syn wt)

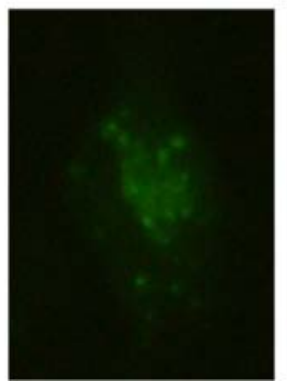

PC12 (Syn $\left.{ }^{\mathrm{mt}}\right)$

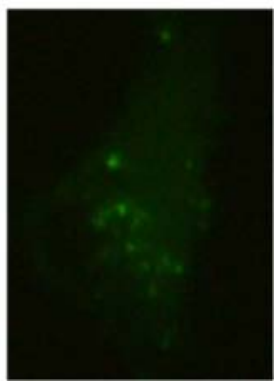

Fig. 2 Levels of autophagy-associated markers and autophagic vesicles induced by starvation in the PC12, PC12 (Con), PC12 (Syn ${ }^{\text {wt }}$ ) or PC12 (Syn ${ }^{\mathrm{mt}}$ ) cells. a: Western blotting assay of LC3-I, LC3-II, Atg 7 and mTOR in the PC12, PC12 (Con), PC12 (Syn ${ }^{\text {wt }}$ ) or PC12 (Syn ${ }^{\mathrm{mt}}$ ) cells post an incubation at $37^{\circ} \mathrm{C}$ in FBS-free medium for 24 hours; $\mathbf{b}$ : Ratio of LC3-II to LC3-I in the starvation-treated cell lines; $\mathbf{c}$ : Relative level of Atg 7 and mTOR levels to -actin in each cell line post the starvation treatment; $\mathbf{d}$ and $\mathbf{e}$ : Imaging (d) and counting (e) of EGFP-positive autophagic vesicles in the cytosol of starvation-treated PC12, PC12 (Con), PC12 (Syn ${ }^{\text {wt }}$ ) or PC12 (Syn ${ }^{\mathrm{mt}}$ ) cells which were transfected with pCDNA3.1-EGFP-LC3 plasmid for another 24 hours; Data was averaged for triple independent results. ${ }^{*} p<0.05,{ }^{* *} p<0.01$, ns: no significance

that the overexpressed HMGB1 promoted more autophagic vesicles in either PC12 $\left(\mathrm{Syn}^{\mathrm{wt}}\right)$ or PC12 $\left(\mathrm{Syn}^{\mathrm{mt}}\right)$ cells which were subject to starvation. Therefore, HMGB1 promotes $\alpha$ synuclein degradation and ameliorates the $\alpha$-synucleinmediated autophagy reduction in PC12 cells.

In addition, we also investigated the influence of exogenous HMGB1 treatment on the starvation-induced autophagy in PC12 cells. Western blotting assay (Fig. 4a) demonstrated that the treatment with 0.2 to $1 \mathrm{~g} / \mathrm{mL}$ HMGB1 did not significantly regulate the conversion of
LC3-I to LC3-II (Fig. 4b) and the expression of Atg 7 (Fig. 4c) and mTOR (Fig. 4d). Therefore, the exogenous HMGB1 exerts no regulation on the starvation-induced autophagy in PC12 cells.

\section{Beclin 1 overexpression promotes autophagy and} a-synuclein degradation in the a-synuclein-overexpressed PC12 cells

Given the high importance of Beclin1-dependent autophagy in the $\alpha$-synuclein degradation $[10,12]$, we then 

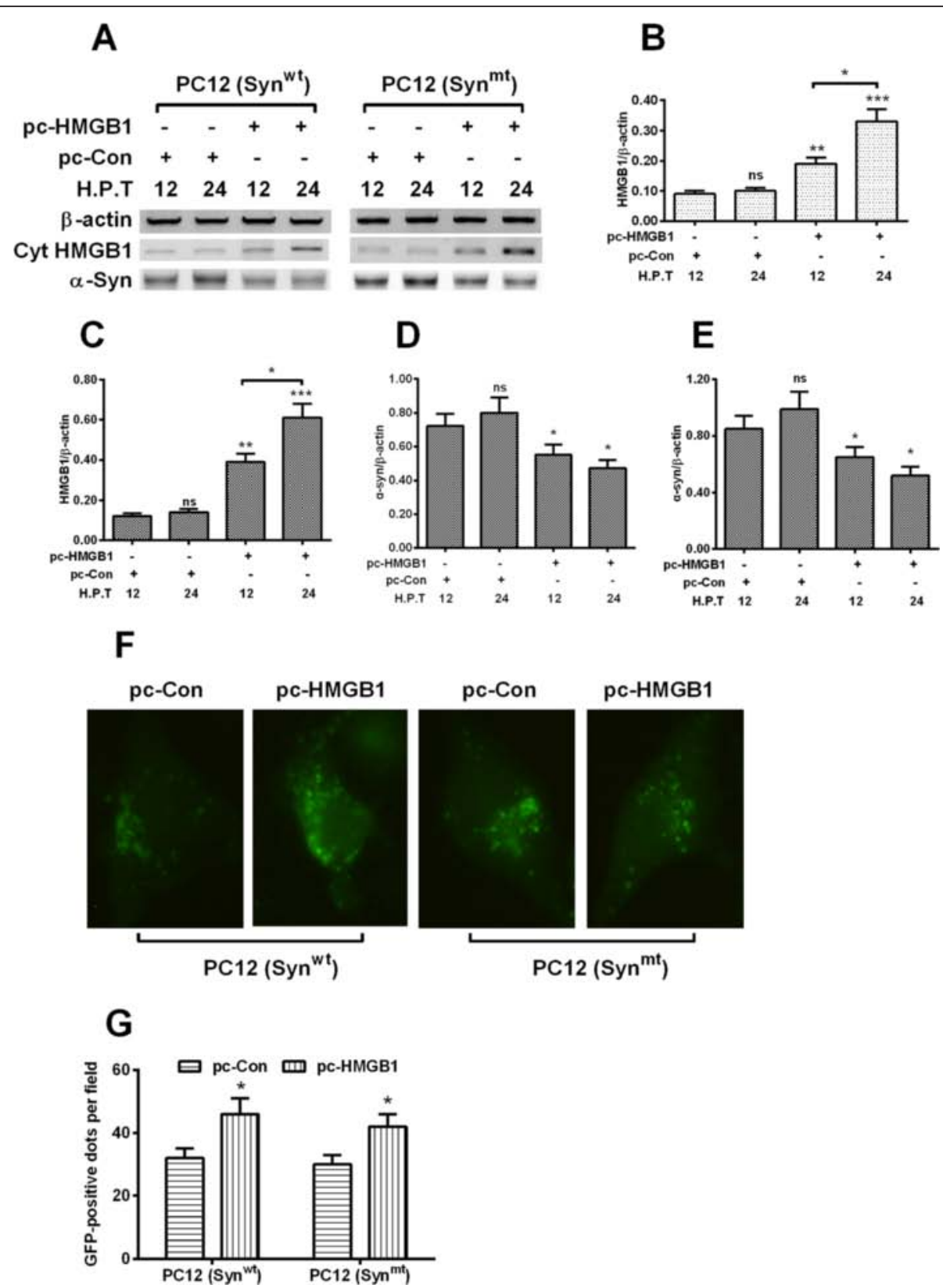

Fig. 3 HMGB1 upregulation inhibits a-synuclein accumulation and ameliorates the a-synuclein-inhibited autophagy in PC12 (Syn ${ }^{\text {wt }}$ ) and PC12 $\left(\right.$ Syn $^{\mathrm{mt}}$ ) cells. a: Western blot analysis of HMGB1 and a-synuclein in PC12 (Syn ${ }^{\text {wt }}$ ) and PC12 (Syn ${ }^{\mathrm{mt}}$ ) cells, which were transfected with HMGB1-pcDNA3.1 (+) or RFP-pcDNA3.1 (+) plasmid for 12 or 24 hours; $\mathbf{b}$ and $\mathbf{c}$ : Ratio of HMGB1 to -actin in PC12 (Syn ${ }^{\text {wt }}$ ) and PC12 (Syn ${ }^{\text {mt }}$ ) cells with or without HMGB1 promoted; $\mathbf{d}$ and e: Ratio of a-synuclein to -actin in PC12 (Syn ${ }^{\text {wt }}$ ) and PC12 (Syn ${ }^{\text {mt }}$ ) cells, with or without HMGB1 promoted; $\mathbf{f}$ and $\mathbf{g}$ : Imaging (f) and counting ( $\mathbf{g})$ of EGFP-positive autophagic vesicles in the cytosol of starvation-treated PC12 (Syn ${ }^{\text {wt }}$ ) or PC12 (Syn ${ }^{\mathrm{mt}}$ ), with or without HMGB1 promoted. Each result was averaged for triple independent experiments. Statistical significance was presented as ${ }^{*} p<0.05$, ${ }^{* *} p<$ $0.01,{ }^{* *} p<0.001$, ns: no significance

investigated the influence of Beclin1 overexpression on the $\alpha$-synuclein degradation and the starvation-induced autophagy in both PC12 $\left(\mathrm{Syn}^{\mathrm{wt}}\right)$ and PC12 $\left(\mathrm{Syn}^{\mathrm{mt}}\right)$ cells. As shown in Fig. 5a, the lentivirus-mediated Beclin1 overexpression in protein level was significant in both cell lines (Fig. $5 \mathrm{~b}$ and $\mathrm{c})$ at either 12 or 24 H.P.I. $(p<$
0.001 respectively) with 1 multiplicity of infection (MOI) with the Beclin1-lentivirus (LV-Beclin1). And the $\alpha$ synuclein level was markedly reduced at 12 or 24 H.P.I. in the LV-Beclin1-infected PC12 (Syn ${ }^{\mathrm{wt}}$ ) (either $p<0.05$ for 12 or 24 H.P.I., Fig. 5 d) or PC12 $\left(\mathrm{Syn}^{\mathrm{mt}}\right)$ cells $(p<$ 0.01 or $p<0.001$ for 12 or 24 H.P.I., Fig. 5e). In addition, 


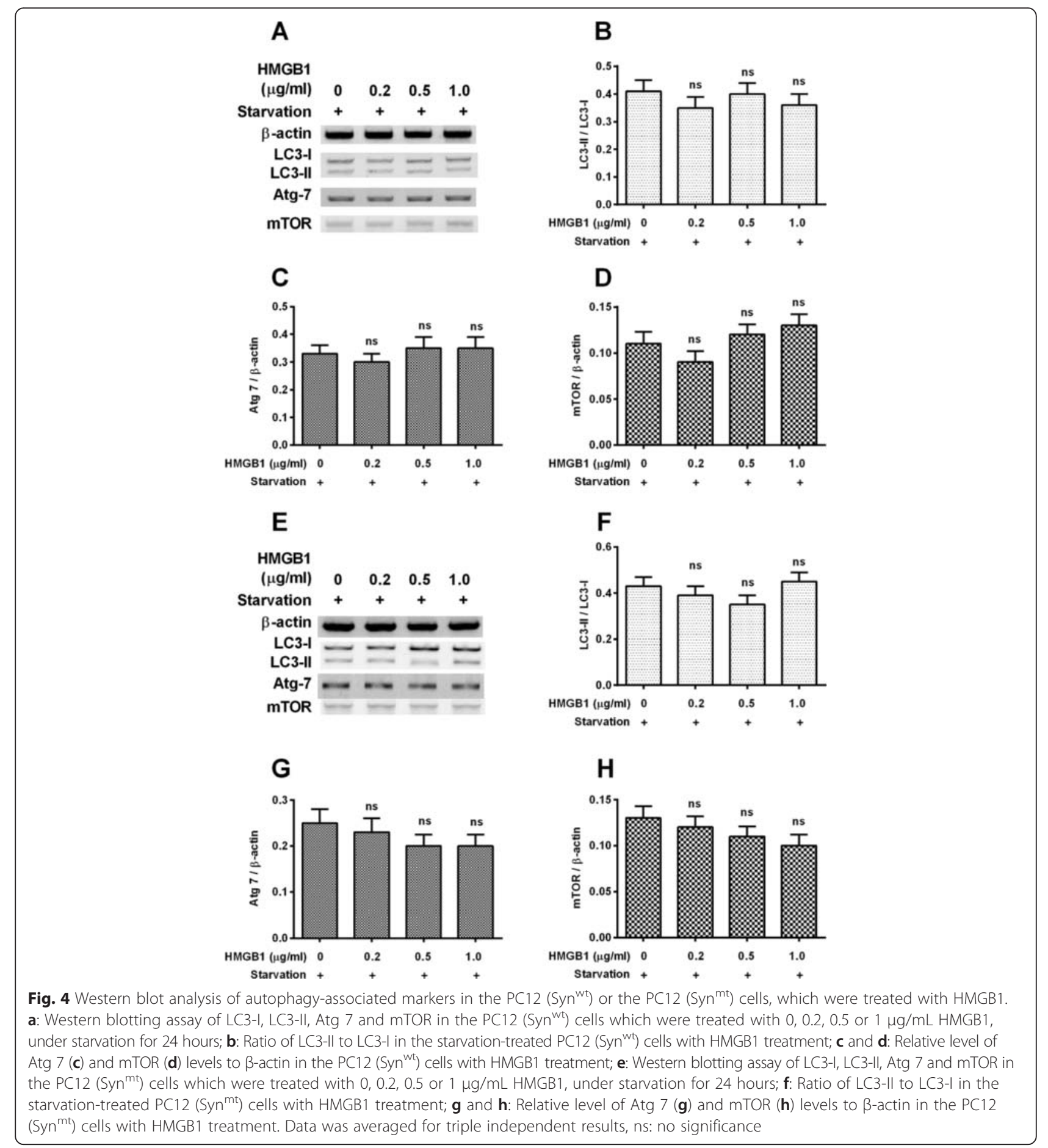

we examined the starvation-induced autophagic vesicles in each cell line with the EGFP-LC3 reporter assay. It was indicated in Fig. $5 \mathrm{f}$ and $\mathrm{g}$ that the infection with 1 MOI LV-Beclin1 promoted more autophagic vesicles than the LV-Con infection in either PC12 $\left(\mathrm{Syn}^{\mathrm{wt}}\right)$ or PC12 $\left(\right.$ Syn $\left.^{\mathrm{mt}}\right)$ cells $(p<0.05)$. Thus, we also confirmed the positive regulation by Beclin1 on the starvationinduced autophagy in PC12 cells.

\section{Discussion}

HMGB1, as a cytokine-like factor [33], is increasingly recognized as a novel autophagy regulator via interfering with the PIK3C3 complex [34]. Nucleus-to-cytosol translocated HMGB1 competitively binds to Beclin1 and subsequently induces autophagy [35]. However, $\alpha$-synuclein has been shown to impair the autophagy [36, 37]. In particular, $\alpha$ synuclein could bind to both cytoplasmic and nuclear 

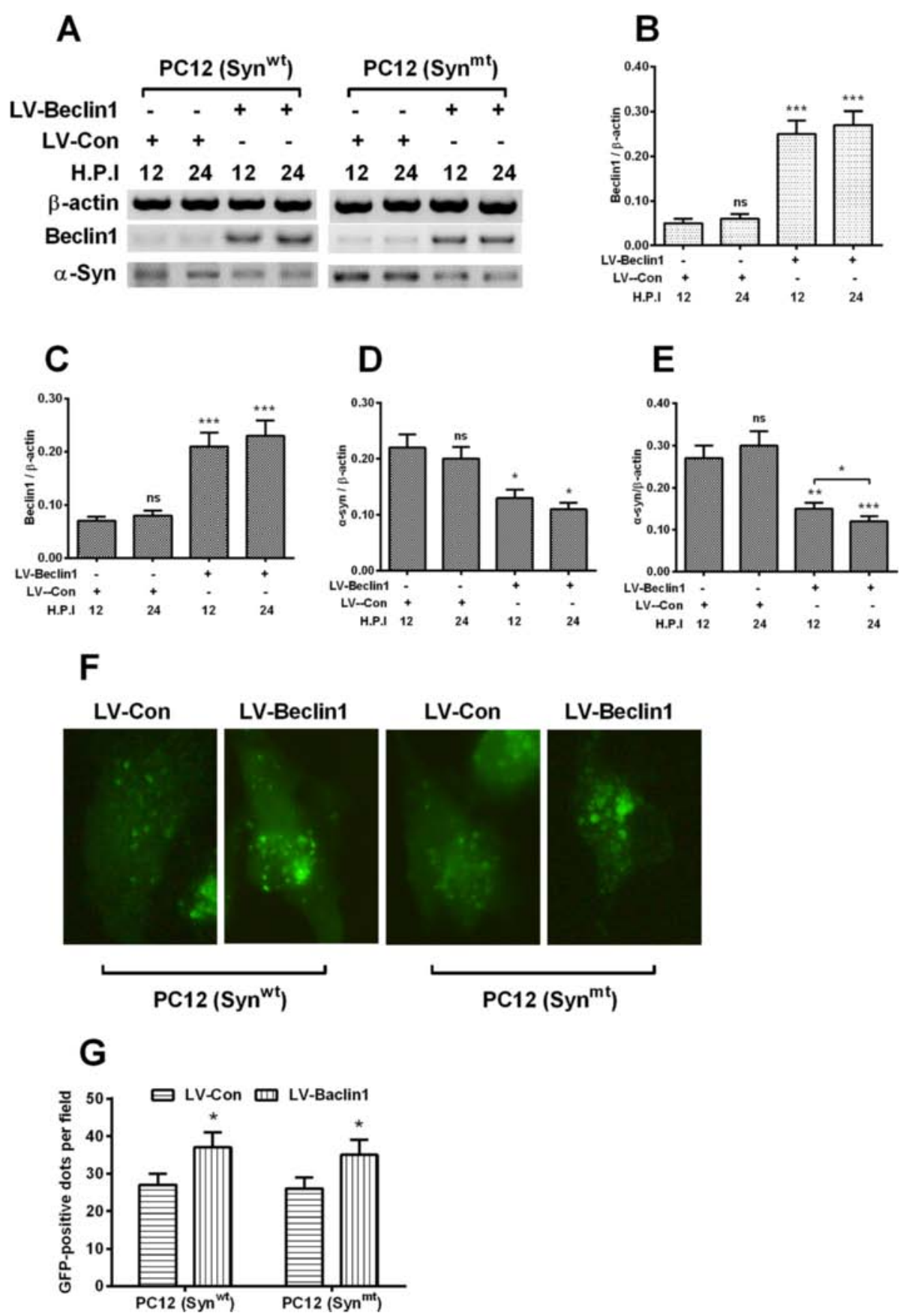

Fig. 5 Beclin 1 upregulation reduces a-synuclein accumulation and ameliorates the a-synuclein-inhibited autophagy in PC12 (Syn $\left.{ }^{w t}\right)$ and PC12 $\left(S y n^{\mathrm{mt}}\right)$ cells. a: Western blot analysis of Beclin1 and a-synuclein in the PC12 (Syn $\left.{ }^{\text {wt }}\right)$ and PC12 (Syn $\left.{ }^{\mathrm{mt}}\right)$ cells which were infected with 1 multiplicity of infection (MOI) pLenti-Beclin1 (LV-Beclin1) or pLenti-Con (LV-Con) under starvation for 12 or 24 hours; $\mathbf{b}$ and $\mathbf{c}$ : Ratio of Beclin1 to -actin in PC12 $\left(S_{y n}{ }^{w t}\right)(\mathbf{b})$ and PC12 (Syn $\left.{ }^{\mathrm{mt}}\right)$ (c) cells which were infected with LV-Beclin1 or with LV-Con virus; $\mathbf{d}$ and e: Ratio of a-synuclein to -actin in PC12 (Syn ${ }^{\text {wt) }}$ (b) and PC12 (Syn ${ }^{\mathrm{mt}}$ ) (c) cells which were infected with LV-Beclin1 or with LV-Con virus; $\mathbf{f}$ and $\mathbf{g}$ : Imaging (f) and counting (g) of EGFP-positive autophagic vesicles in the starvation-treated PC12 (Syn $\left.{ }^{w t}\right)$ or PC12 (Syn ${ }^{\mathrm{mt}}$ ), which were infected with LV-Beclin 1 or with LV-Con virus. Each result was averaged for triple independent experiments. Statistical significance was presented as ${ }^{*} p<0.05,{ }^{* *} p<0.01,{ }^{* * *} p<0.001$, ns: no significance

HMGB1, block HMGB1-Beclin1 binding, whereas to strengthen the Beclin1-BcL2 binding [31], and thus to inhibit autophagy. And such inhibition could be restored by the Beclin 1 overexpression. In the present study, we found that the overexpression of either WT or MT $\alpha$ synuclein markedly reduced the cytoplasmic levels of both 
HMGB1 and Beclin1 in PC12 cells, implying the reduced HMGB1 and Beclin1 might contribute to the $\alpha$-synucleinmediated autophagy inhibition in PC12 cells.

Impaired autophagy has been indicated to correlate with the $\alpha$-synuclein aggregation and neurodegeneration in PD [38-41]; and the stimulated autophagy could reduce the accumulation of $\alpha$-synuclein in cells and in mouse brain [12, 42], and could even rescue midbrain dopamine neurons from $\alpha$-synuclein toxicity [43]. On the other side, $\alpha$-synuclein has been shown to impair autophagy [36, 37]. The overexpression of either WT or MT $\alpha$-synuclein inhibits autophagy in pheochromocytoma PC12 cells [31], impairs neurite outgrowth of primary midbrain neurons, affects neurite branching [44]. In this study, we reconfirmed such autophagy inhibition by $\alpha$-synuclein overexpression in PC12 cells, the overexpression of wild-type or mutant-type $\alpha$-synuclein significantly downregulated the starvation-induced autophagy via inhibiting the mTOR/Atg 7 signaling. Therefore, we also confirmed the autophagy inhibition by the overexpressed $\alpha$-synuclein in PC12 cells. And it implies that the reduced HMGB1 and Beclin1 might contribute to the reduced autopahgy in the $\alpha$-synuclein-overexpressed PC12 cells.

HMGB1 is also known as the high-mobility group protein 1 (HMG-1). As a chromatin-associated nuclear protein, it is a critical regulator of autophagy. And the pharmacological inhibition of HMGB1 cytoplasmic translocation limits starvation-induced autophagy. Moreover, only endogenous HMGB1 has been indicated to regulate the Bcl-2-Beclin1 binding, via the direct interact with Beclin 1 [34, 35], and thus regulating the convergence of autophagy and apoptosis, via interacting with antiapoptotic Bcl-2-like proteins [45]. Our study confirmed that either endogenous HMGB1 or Beclin 1 overexpression promoted the $\alpha$-synuclein degradation in $\mathrm{PC} 12$ cells. Moreover, the overexpressed HMGB1 or Beclin 1 markedly ameliorated the $\alpha$-synuclein-mediated autophagy reduction in the $\alpha$-synuclein-overexpressed (either WT or MT) PC12 cells, implying the promotion by HMGB1 to the $\alpha$-synuclein degradation might be autophagy-dependent. And such amelioration might be dependent on the HMGB1-Beclin1 interaction.

Interesting, our results indicated that the extracellular HMGB1 exerted no regulatory role on the starvationinduced autophagy, posing no influence on the levels of mTOR and Atg 7 in both PC12 (Syn ${ }^{\mathrm{wt}}$ ) and PC12 $\left(\mathrm{Syn}^{\mathrm{mt}}\right)$ cells. HMGB1 is a secretary cytokine from activated macrophages and monocytes [46] to mediate inflammation [33], via binding to receptor for advanced glycation endproducts (RAGE) [47] or to toll-like receptor (TLR) [48]. However, the present study confirmed that the promotion by HMGB1 was not RAGE- or TLRdependent. In addition, the gain-of-function strategy also confirmed the promotion to the $\alpha$-synuclein degradation and the autophagy induction by Beclin1 overexpression in PC12 cells. Taken together, we speculated that endogenous HMGB1 and Beclin1 might promote the autophagic degradation of $\alpha$-synuclein. Therefore, the endogenous HMGB1 and Beclin1 exerts protective role in the cells against the $\alpha$-synuclein accumulation.

\section{Conclusion}

In summary, $\alpha$-synuclein with wild-type or mutant-type inhibited autophagy in PC12 cells via inhibiting the HMGB1 and Beclin1. On the other side, the cytosolic promotion to HMGB1 or to Beclin1 up-regulates the autophagic degradation of $\alpha$-synuclein via increasing the autophagy in PC12 cells. Therefore, the endogenous HMGB1 and Beclin1 present protective role in the cells against the $\alpha$-synuclein accumulation.

\section{Abbreviations}

PD: Parkinson disease; HMGB1: High mobility group box 1; ALP: Autophagy lysosomal pathway; WT: Wild-type; (MT: A53T and A30P), Mutant-type;

ULK1: Unc-51 like autophagy activating kinase 1; mTOR: Mammalian target of rapamycin; PIK3C3: Beclin 1-Phosphatidylinositol 3-kinase catalytic subunit type 3; ATG: Autophagy-related protein; VPS34: Vacuolar protein sorting 34; ROCK1: Rho-associated, coiled-coil containing protein kinase 1; Mst1: Macrophage stimulating 1; PC12 (Syn ${ }^{\mathrm{mt}}$ ): PC12 cell overexpressing MT (A53T and A30P) a-synuclein; PC12 (Syn ${ }^{\text {wt }}$ ): PC12 cell overexpressing WT a-synuclein.

\section{Competing interests}

Authors declare no conflict of interests regarding the publication of this article.

\section{Authors' contributions}

$\mathrm{KHW}, \mathrm{JMH}$ and $\mathrm{WX}$ designed the research, $\mathrm{KHW}, \mathrm{JMH}, \mathrm{LJH}, \mathrm{CHZ}$ and $\mathrm{ZZC}$ performed the experiments. KHW, JMH and WX conceived of the study, drafted the manuscript. KHW and ZZC performed the statistical analysis. All authors read and approved the final manuscript.

\section{Acknowledgements}

The present study was supported by the grant from the Science and Technology Project from Guangxi Science and Technology Bureau (GKG1355005-4-3).

\section{Author details}

'Department of Neurology, RuiKang Hospital Affiliated to Guangxi University of Chinese Medicine, Nanning, Guangxi 530011, China. ${ }^{2}$ Southern Medical University, Guangzhou, Guangdong 510515, China. ${ }^{3}$ Department of

Traditional Chinese Medicine, Nanfang Hospital, Southern Medical University, Guangzhou, Guangdong 510515, China. ${ }^{4}$ School of Traditional Chinese Medicine, Southern Medical University, Guangzhou, Guangdong 510515, China. ${ }^{5}$ Guangxi University of Chinese Medicine, Nanning, Guangxi 530001, China.

\section{Received: 10 October 2015 Accepted: 14 January 2016} BW

\section{References}

1. Irizarry MC, Growdon W, Gomez-Isla T, Newell K, George JM, Clayton DF, et al. Nigral and cortical Lewy bodies and dystrophic nigral neurites in Parkinson's disease and cortical Lewy body disease contain alpha-synuclein immunoreactivity. J Neuropathol Exp Neurol. 1998;57(4):334-7.

2. Spillantini MG, Crowther RA, Jakes R, Hasegawa M, Goedert M. alpha-Synuclein in filamentous inclusions of Lewy bodies from Parkinson's disease and dementia with lewy bodies. Proc Natl Acad Sci U S A. 1998;95(11):6469-73.

3. Ebrahimi-Fakhari D, McLean PJ, Unni VK. Alpha-synuclein's degradation in vivo: opening a new (cranial) window on the roles of degradation pathways in Parkinson disease. Autophagy. 2012;8(2):281-3. 
4. Ebrahimi-Fakhari D, Wahlster L, McLean PJ. Protein degradation pathways in Parkinson's disease: curse or blessing. Acta Neuropathol. 2012;124(2):153-72.

5. Shacka JJ, Roth KA, Zhang J. The autophagy-lysosomal degradation pathway: role in neurodegenerative disease and therapy. Front Biosci. 2008;13:718-36.

6. Pan T, Kondo S, Le W, Jankovic J. The role of autophagy-lysosome pathway in neurodegeneration associated with Parkinson's disease. Brain. 2008;131(Pt 8): 1969-78.

7. Ding $W X$, Yin $X M$. Sorting, recognition and activation of the misfolded protein degradation pathways through macroautophagy and the proteasome. Autophagy. 2008;4(2):141-50.

8. Cook C, Stetler C, Petrucelli L. Disruption of protein quality control in Parkinson's disease. Cold Spring Harb Perspect Med. 2012;2(5):a9423.

9. Ravikumar B, Sarkar S, Davies JE, Futter M, Garcia-Arencibia M, Green-Thompson ZW, et al. Regulation of mammalian autophagy in physiology and pathophysiology. Physiol Rev. 2010;90(4):1383-435.

10. Spencer B, Potkar R, Trejo M, Rockenstein E, Patrick C, Gindi R, et al. Beclin 1 gene transfer activates autophagy and ameliorates the neurodegenerative pathology in alpha-synuclein models of Parkinson's and Lewy body diseases. J Neurosci. 2009;29(43):13578-88.

11. Wong PM, Feng Y, Wang J, Shi R, Jiang X. Regulation of autophagy by coordinated action of mTORC1 and protein phosphatase 2A. Nat Commun. 2015;6:8048

12. Chen LL, Song JX, Lu JH, Yuan ZW, Liu LF, Durairajan SS, et al. Corynoxine, a natural autophagy enhancer, promotes the clearance of alpha-synuclein via Akt/mTOR pathway. J Neuroimmune Pharmacol. 2014:9(3):380-7.

13. Moreau K, Ghislat G, Hochfeld W, Renna M, Zavodszky E, Runwal G, et al. Transcriptional regulation of Annexin A2 promotes starvation-induced autophagy. Nat Commun. 2015;6:8045.

14. Ma B, Cao W, Li W, Gao C, Qi Z, Zhao Y, et al. Dapper1 promotes autophagy by enhancing the Beclin1-Vps34-Atg14L complex formation. Cell Res. 2014; 24(8):912-24.

15. Zhao J, Wang ML, Li Z, Gao DM, Cai Y, Chang J, et al. Interferon-alpha-2b induces autophagy in hepatocellular carcinoma cells through Beclin1 pathway. Cancer Biol Med. 2014;11(1):64-8.

16. Gurkar AU, Chu K, Raj L, Bouley R, Lee SH, Kim YB, et al. Identification of ROCK1 kinase as a critical regulator of Beclin1-mediated autophagy during metabolic stress. Nat Commun. 2013;4:2189.

17. Maejima Y, Kyoi S, Zhai P, Liu T, Li H, Ivessa A, et al. Mst1 inhibits autophagy by promoting the interaction between Beclin1 and Bcl-2. Nat Med. 2013; 19(11):1478-88.

18. Pan CC, Kumar S, Shah N, Bloodworth JC, Hawinkels $\sqcup$, Mythreye K, et al. Endoglin Regulation of Smad2 Function Mediates Beclin1 Expression and Endothelial Autophagy. J Biol Chem. 2015;290(24):14884-92.

19. Sun $X$, Tang D. HMGB1-dependent and -independent autophagy. Autophagy. 2014;10(10):1873-6.

20. Huang J, Ni J, Liu K, Yu Y, Xie M, Kang R, et al. HMGB1 promotes drug resistance in osteosarcoma. Cancer Res. 2012;72(1):230-8.

21. Luo Y, Yoneda J, Ohmori H, Sasaki T, Shimbo K, Eto S, et al. Cancer usurps skeletal muscle as an energy repository. Cancer Res. 2014;74(1):330-40.

22. Perez-Carrion MD, Cena V. Knocking down HMGB1 using dendrimerdelivered siRNA unveils its key role in NMDA-induced autophagy in rat cortical neurons. Pharm Res. 2013;30(10):2584-95.

23. Hagiwara S, Iwasaka H, Hasegawa A, Kudo K, Kusaka J, Oyama Y, et al. Infusion of a glucose solution reduces autophagy in the liver after LPSinduced systemic inflammation. Inflammation. 2012;35(1):249-58.

24. Fang H, Liu A, Dahmen U, Dirsch O. Dual role of chloroquine in liver ischemia reperfusion injury: reduction of liver damage in early phase, but aggravation in late phase. Cell Death Dis. 2013;4:e694.

25. Monahan AJ, Warren M, Carvey PM. Neuroinflammation and peripheral immune infiltration in Parkinson's disease: an autoimmune hypothesis. Cell Transplant. 2008;17(4):363-72

26. Hirsch EC, Hunot S. Neuroinflammation in Parkinson's disease: a target for neuroprotection? Lancet Neurol. 2009:8(4):382-97.

27. Przedborski S. Inflammation and Parkinson's disease pathogenesis. Mov Disord. 2010;25 Suppl 1:S55-7.

28. Block ML, Hong JS. Chronic microglial activation and progressive dopaminergic neurotoxicity. Biochem Soc Trans. 2007;35(Pt 5):1127-32.

29. Zhu X, Messer JS, Wang Y, Lin F, Cham CM, Chang J, et al. Cytosolic HMGB1 controls the cellular autophagy/apoptosis checkpoint during inflammation. J Clin Invest. 2015;125(3):1098-110.
30. Gao HM, Zhou H, Zhang F, Wilson BC, Kam W, Hong JS. HMGB1 acts on microglia Mac1 to mediate chronic neuroinflammation that drives progressive neurodegeneration. J Neurosci. 2011;31(3):1081-92.

31. Song JX, Lu JH, Liu LF, Chen LL, Durairajan SS, Yue Z, et al. HMGB1 is involved in autophagy inhibition caused by SNCA/alpha-synuclein overexpression: a process modulated by the natural autophagy inducer corynoxine B. Autophagy. 2014;10(1):144-54.

32. Schmittgen TD, Livak KJ. Analyzing real-time PCR data by the comparative C(T) method. Nat Protoc. 2008;3(6):1101-8.

33. Wang $\mathrm{H}$, Bloom $\mathrm{O}$, Zhang $\mathrm{M}$, Vishnubhakat JM, Ombrellino $\mathrm{M}$, Che J, et al. HMG-1 as a late mediator of endotoxin lethality in mice. Science. 1999; 285(5425):248-51

34. Kang R, Livesey KM, Zeh HJ, Loze MT, Tang D. HMGB1: a novel Beclin 1-binding protein active in autophagy. Autophagy. 2010;6(8):1209-11.

35. Tang D, Kang R, Livesey KM, Cheh CW, Farkas A, Loughran P, et al. Endogenous HMGB1 regulates autophagy. J Cell Biol. 2010;190(5):881-92.

36. Winslow AR, Rubinsztein DC. The Parkinson disease protein alpha-synuclein inhibits autophagy. Autophagy. 2011;7(4):429-31.

37. Ferrucci M, Pasquali L, Ruggieri S, Paparelli A, Fornai F. Alpha-synuclein and autophagy as common steps in neurodegeneration. Parkinsonism Relat Disord. 2008;14 Suppl 2:S180-4.

38. Plotegher $\mathrm{N}$, Civiero L. Neuronal autophagy, alpha-synuclein clearance, and LRRK2 regulation: a lost equilibrium in parkinsonian brain. J Neurosci. 2012; 32(43):14851-3.

39. Friedman LG, Lachenmayer ML, Wang J, He L, Poulose SM, Komatsu M, et al. Disrupted autophagy leads to dopaminergic axon and dendrite degeneration and promotes presynaptic accumulation of alpha-synuclein and LRRK2 in the brain. J Neurosci. 2012;32(22):7585-93.

40. Malkus KA, Ischiropoulos $H$. Regional deficiencies in chaperone-mediated autophagy underlie alpha-synuclein aggregation and neurodegeneration. Neurobiol Dis. 2012:46(3):732-44.

41. Li G, Yang H, Zhu D, Huang H, Liu G, Lun P. Targeted suppression of chaperone-mediated autophagy by miR-320a promotes alpha-synuclein aggregation. Int J Mol Sci. 2014;15(9):15845-57.

42. Steele JW, Ju S, Lachenmayer ML, Liken J, Stock A, Kim SH, et al. Latrepirdine stimulates autophagy and reduces accumulation of alphasynuclein in cells and in mouse brain. Mol Psychiatry. 2013;18(8):882-8.

43. Decressac M, Mattsson B, Weikop P, Lundblad M, Jakobsson J, Bjorklund A. TFEB-mediated autophagy rescues midbrain dopamine neurons from alphasynuclein toxicity. Proc Natl Acad Sci U S A. 2013;110(19):E1817-26.

44. Koch JC, Bitow F, Haack J, D'Hedouville Z, Zhang JN, Tonges L, et al. Alpha-Synuclein affects neurite morphology, autophagy, vesicle transport and axonal degeneration in CNS neurons. Cell Death Dis. 2015;6:e1811.

45. Liang $X H$, Kleeman LK, Jiang HH, Gordon G, Goldman JE, Berry G, et al. Protection against fatal Sindbis virus encephalitis by beclin, a novel $\mathrm{BCl}-2$ interacting protein. J Virol. 1998;72(11):8586-96.

46. Klune JR, Dhupar R, Cardinal J, Billiar TR, Tsung A. HMGB1: endogenous danger signaling. Mol Med. 2008;14(7-8):476-84.

47. Sims GP, Rowe DC, Rietdijk ST, Herbst R, Coyle AJ. HMGB1 and RAGE in inflammation and cancer. Annu Rev Immunol. 2010;28:367-88.

48. Yu M, Wang H, Ding A, Golenbock DT, Latz E, Czura CJ, et al. HMGB1 signals through toll-like receptor (TLR) 4 and TLR2. Shock. 2006;26(2):174-9.

\section{Submit your next manuscript to BioMed Central and we will help you at every step:}

- We accept pre-submission inquiries

- Our selector tool helps you to find the most relevant journal

- We provide round the clock customer support

- Convenient online submission

- Thorough peer review

- Inclusion in PubMed and all major indexing services

- Maximum visibility for your research

Submit your manuscript at www.biomedcentral.com/submit 